\title{
Study on the Influence Factors of the Luminous Intensity of the Long Afterglow Luminous Paints
}

\author{
ZHAO Su, WEI Wei, WU Qian and GUAN Daren \\ School of Material Science and Engineering, Shenyang Jianzhu University, Shenyang 110168, \\ China \\ zhaosu2005@126.com¹,374546440@qq.com,718673265@qq.com,375533139@qq.com
}

\begin{abstract}
In order to extend the time afterglow luminous powder, enhancement the brightness of luminous paint, this study explore affect long afterglow energy storage luminous paints brightness of the main factors. Luminous paints were prepared with rare earth aluminate long afterglow luminescent powder, first is luminous powder surface modification, then investigate the influence of light emitting powder content, calcium carbonate, titanium dioxide, nano alumina and other fillers on the luminescent properties of the paints. It was concluded that the water resistance of the luminescent powder is better and the brightness can be improved after the modification of anhydrous alcohol. The addition of nano-alumina can improve the brightness of the system, and can effectively enhance the hardness of the paints. In the paints, the two kinds of components of carbonate and titanium dioxide have little effect on the luminescent brightness of the painting.
\end{abstract}

\section{Introduction}

Long afterglow luminous powder is a new type of functional materials, it can absorb light energy and store it. After a period, the stored energy will be released in the form of light, then used for safety instructions and lighting ${ }^{[1-3]}$.Long afterglow luminous paint basically is made of long afterglow luminous powder and emulsion, adding in fillers and additives according to a certain ration. Why the paint can light? It depends on luminous powder, so the research process of luminous paint is based on the luminous powder.

U.S. Patent US359048 describes a colored luminous paint ${ }^{[4]}$, the main component is an alkyd resin binder and alumina earth phosphors, and added with other additives. Another U.S. Patent US5472737 developed a kind of luminous paint used for highway, the main ingredient come from polyurethane resin of the modified acrylic. Japan developed a luminous paint, the painting is made of high purity alumina and rare earth elements mixed by high temperature calcination, added a certain of resin and additives. In short, some developed countries already have a more mature technology of energy storage luminous paint.

There are also many Chinese scholars have made certain achievements in the field of luminous paint. Ai Hua Yang ${ }^{[5]}$ research on the wall luminous paint. The luminescent paint

* Corresponding author: zhaosu2005@126.com 
is an rare earth aluminate luminescent material, based on modified silicone-acrylic resin, and added a right amount of resin and additives, this luminous paint is a kind of solvent based paints. Ningwen Fang developed a nighttime luminous paint, consists of luminous powder, resin, rubber and so on, widely used in signage, decoration and other fields. Luminous paint has not been widely used in China as other developed countries since luminous powder is costly and afterglow time is too short. The purpose of this study is to extend the time for afterglow luminescent power and enhance brightness for paintings.

\section{Experimental}

\subsection{Experimental Procedure}

1) According to take $10 \mathrm{~g}$ luminescent power in $50 \mathrm{~g}$, then add adequate amount of the dispersing agent and multifunction additive, stir for $30 \mathrm{~min}$ while the temperatures hover around 60 degrees. The samples will be put in several minutes, a quick modified luminescent power gotten.

2) Put dispersant and sectional dofonmer into deionized water under low speed stirring conditions, then followed by adding luminous powder.

3) Increasing the stirring speed, then mixed thickeners, leveling agents, film-forming additives in system.

4) After uniformly dispersed for 20-30 minutes, reduce the speed then slowly added styrene-acrylic emulsion and the remaining defoamer, and adjust the PH value of 8 to 9 .

5)Finally, use thickeners or water to adjust the viscosity of the paint.

\subsection{Performance Test}

Test method for specified performance of luminous painting is according to GB/T9755-2001 government standard for exterior painting.

\section{Results and Analysis}

\subsection{The Influence of Surface of Luminescent Power}

In this study we choose the rare earth aluminate long afterglow luminescent power which is widely used ,but the biggest shortcoming of this luminescent material is poor moisture resistance .It can be easily hydrolyzed in aqueous or humid environment, and produce aluminum hydroxide precipitation and water-soluble $\mathrm{OH}^{-[6]}$,so phosphor crystal structure will change coming along with system PH raised. Finally, brightness and afterglow time will loss more.

In the case of constant temperature and stirring rate, take four kinds of solvent to surface modified of luminescent power .The experimental results are shown in Figure 1. 


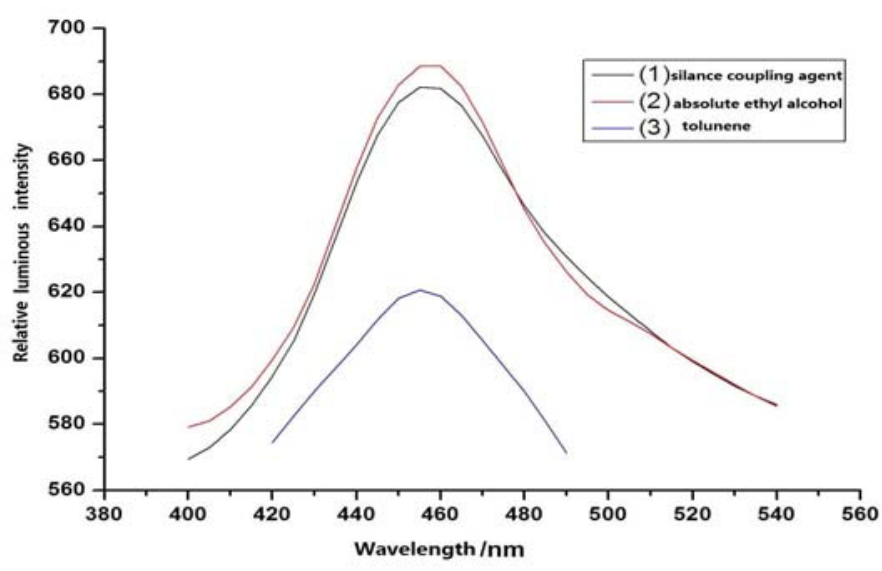

Figure 1.Comparison chart of luminous power brightness

From Figure 1, it can be concluded that the brightest sample is the one which modified by absolute ethyl alcohol, next is silicane coupling agent, tolunene is the last. Modified by absolute ethyl alcohol and silicane had got the peak at the same wavelength, and the peak only had little difference. We chose absolute ethyl alcohol as the solvent for modified luminescent power, the most important thing is the functional group from luminescent power can bonding react with hydroxy from absolute ethyl alcohol, then organic molecules and inorganic particle bond together, the more waterproof luminescent power we have got $^{[7]}$.As the price for absolute ethyl alcohol is very good and most everything ran smoothly.

Used luminous powder before and after modification prepared for luminous paint, photoed by S-4800 emission scanning electron microscope and test results is shown in Figure 2, 3.

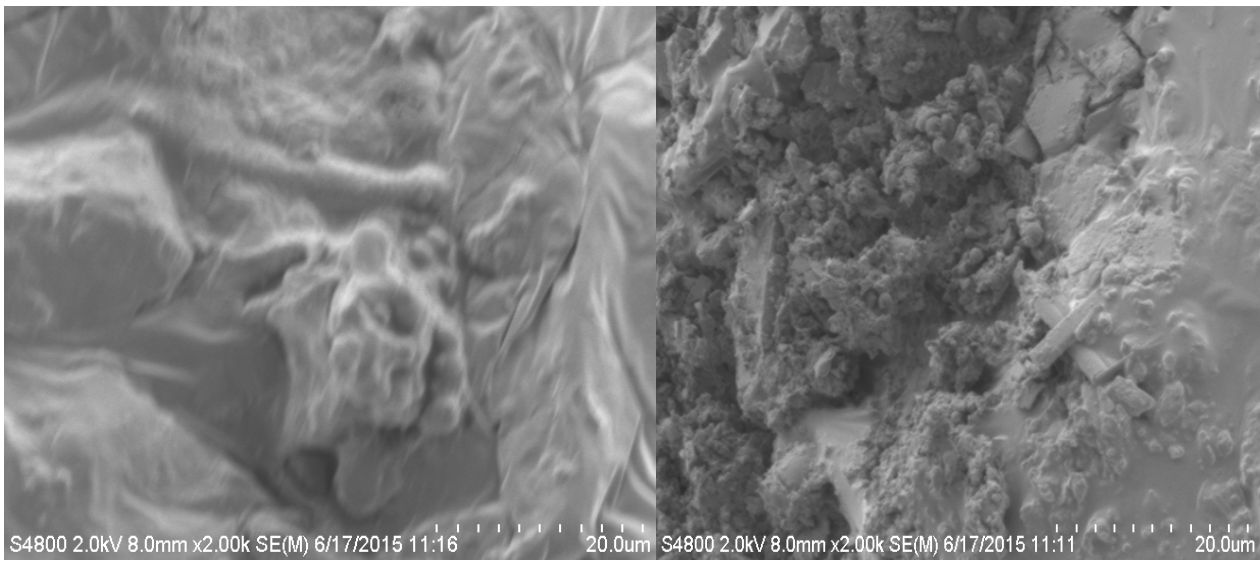

Figure 2. before modification in SEM (left),

Figure 3. after modification in SEM (right)

Figure 2 shows that sample surface smooth before modification, and there is a little pores. But in Figure 3 the modified sample has developed pores in the paint, some of which deep into the interior of the paint. Paint surface is rough and uneven. It can be proved that the water resistance of luminous powder was enhanced, while enhanced it's stability, so the luminous powder can not subjected to hydrolysis reaction. This structure shape makes 
strong brightness for paint, increased it s strength and extend the time for afterglow.

\subsection{The Influence of Content of Luminescent Power}

The second generation of rare earth aluminate luminous powder, the main ingredient is $\mathrm{SrAO}_{4}: \mathrm{Eu}^{2+}, \mathrm{Dy}^{3+}$.In the case of constant temperature and stirring rate, study its effect on brightness by changing the content of luminescent power. The experimental results are shown in Figure 4.

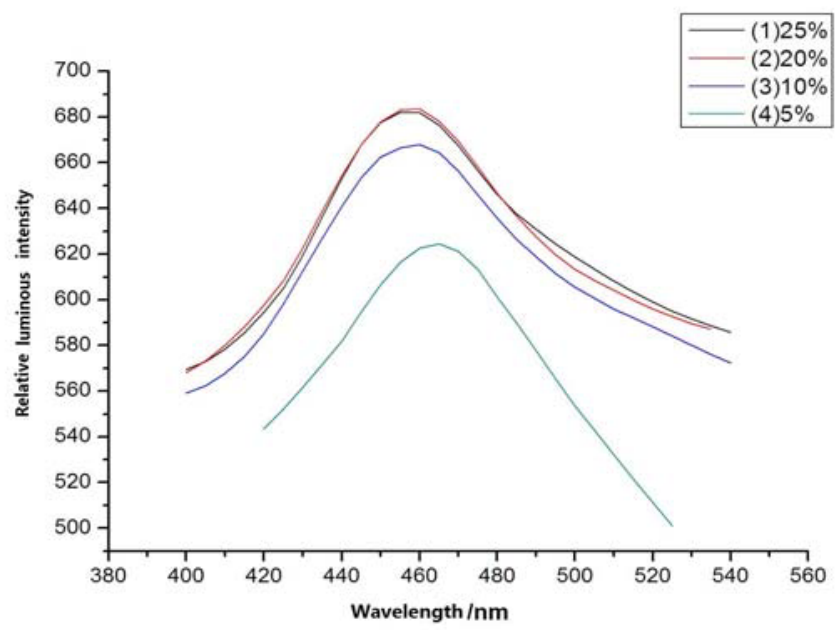

Figure 4.Effect of luminous powder content on the brightness of paints

It can be shown by Figure 4, the paint has the least intensity at the content of $5 \%$, at the content of $10 \%$, the emission intensity was significantly enhanced. When the content increased to $20 \%$, system of emission luminance ratio has enhance more than $10 \%$, and two curves get the peak at the same wavelength of 460 . But content added to $25 \%$, the brightness has no difference with content of $20 \%$,it is because when excessive luminescent power content, make the mutual occlusion between particles, hindering the inner layer of the light-emitting, so it did not achieve effect for enhanced brightness .Shortly, considering the economic costs, as higher prices for luminous powder, so, luminous powder at the content of $20 \%$ is a good choice.

\subsection{The Influence of Content of Nano-alumina}

Test for paint brightness of nano-alumina are shown in Figure 5, 6 . 


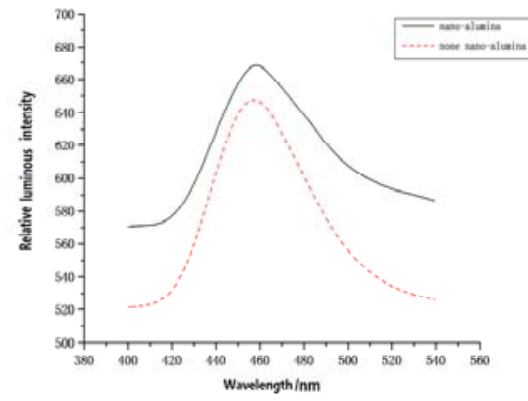

Figure 5. effect in spectral analysis (left),

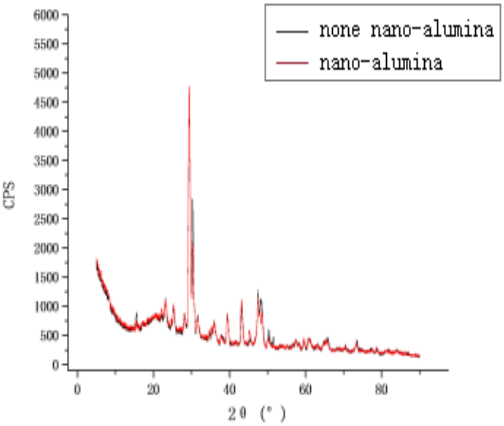

Figure 6. effect in $\mathrm{XRD}$ (right)

According to the results of the Figure 5, the two curves get the peak at the same wavelength of 460 ,With the increasing wavelength at right side of the peak, the relative strength of the painting does not contain nano-alumina is reduced too much. From Figure 6, the peak gotten around $2 \theta=30$, and there are obvious differences between the two peaks. With the nano-alumina was added, it reacts the Al-O-Al bond with highly active hydroxy $l^{[8]}$.Gradually produce the nano-alumina painting layer, make the luminescent power microencapsulation. Thus impeding the hydrolysis reaction of luminescent powder then improve its brightness.

\subsection{The Influence of Content of Calcium Carbonate}

Test for paint brightness of calcium carbonate and titanium dioxide are shown in Figure7.

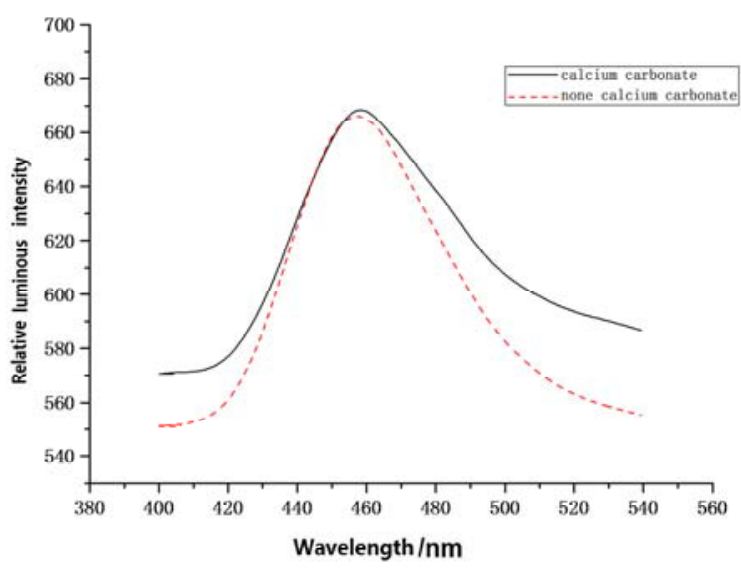

Figure 7. effect of calcium carbonate

It can be seen in Figure 7, there has no effect for whether contains calcium carbonate at the peak. But the paint which contains calcium carbonate is brighter. They have get the peak at the same wavelength of 460 and the paint has more relative intensity the one 
contains calcium at the right side of peak.

\subsection{The Influence of Content of Titanium Dioxide} 8.

Test for paint brightness of calcium carbonate and titanium dioxide are shown in Figure

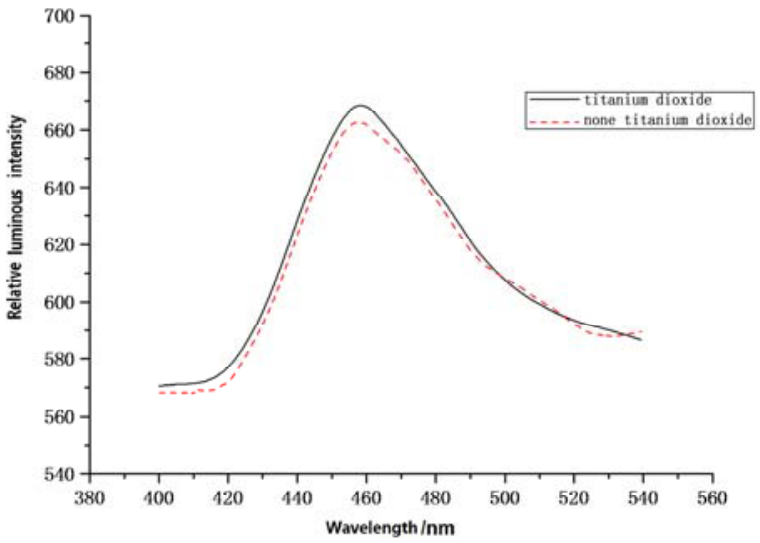

Figure 8. effect of titanium dioxide

According to the results of the Figure 8, itanium dioxide has little effect for the paint, but it's better to keep it .Titanium dioxide in the system have to prevent sagging and cracking effect and enhance water resistance and abrasion resistance of paintings ${ }^{[9]}$,so titanium dioxide is one of the important filler for paint.

\section{Conclusions}

Use $\mathrm{SrAO}_{4}: \mathrm{Eu}^{2+}, \mathrm{Dy}^{3+}$ luminescent powder, styrene-acrylic emulsion and various fillers, additives, prepared for paint. This study discusses the factors that affect brightness for luminous paint.

(1)After modified by absolute ethyl alcohol, water resistance for luminescent powder has increased, PH value decreased. There has more brightness for modified by absolute ethyl alcohol due to preventing the hydrolysis reaction of luminescent powder.

(2)With the increasing content of the luminescent powder, the brightness of luminous paint has enhanced. The best proportion is $20 \%$.

(3)There has no obvious difference for calcium carbonate and titanium dioxide.

(4)Added with nano-alumina, it significantly improve the luminous paint brightness, and improve the painting hardness.

\section{References}

1. Jianfeng Liang, Rongzhi Liu, Zihao Zhou, et al J.China Materials,(2004)10,40-42(In Chinese)

2. Jinhong Zhang, Wukui Zhao, et al J.Plastic Additives,(2006)6,51-52 (In Chinese)

3. YingliangLiu, DingHong, J. Chinese Journal of lnorganic Chemistry, 
(2001)17,181-187(In Chinese)

4. Van Duynhoven. T intabl e Luminescent Paint. 359048. 2002- 03- 19

5. Aihua Yang,Jilin Buliding Materials(2002)214-16(In Chinese)

6. Shengfei Yu,Xiufang Wen,et al J.Paint\&Coatings Industry(2007)3(In Chinese)

7. B K, R H. CN, 1414886, 2003

8. Meixia Li, Luo Ji,et al J.Power Metallurgy Technology(2011) 29(3), 214-217(In Chinese)

9. GaodongXu. FineChemicalIndustrialRawMaterials\&Intermediates(2011)2,23-24(In Chinese) 